\title{
Entropy analysis of human death uncertainty
}

\author{
J. A. Tenreiro Machado • António M. Lopes $\mathbb{i}$
}

Received: 10 February 2021 / Accepted: 27 April 2021 / Published online: 21 May 2021

(C) The Author(s), under exclusive licence to Springer Nature B.V. 2021

\begin{abstract}
Uncertainty about the time of death is part of one's life, and plays an important role in demographic and actuarial sciences. Entropy is a measure useful for characterizing complex systems. This paper analyses death uncertainty through the concept of entropy. For that purpose, the Shannon and the cumulative residual entropies are adopted. The first may be interpreted as an average information. The second was proposed more recently and is related to reliability measures such as the mean residual lifetime. Data collected from the Human Mortality Database and describing the evolution of 40 countries during several decades are studied using entropy measures. The emerging country and inter-country entropy patterns are used to characterize the dynamics of mortality. The locus of the two entropies gives a deeper insight into the dynamical evolution of the human mortality data series.
\end{abstract}

Keywords Entropy $\cdot$ State state portrait $\cdot$ Hierarchical clustering $\cdot$ Lifetable $\cdot$ Death uncertainty

J. A. Tenreiro Machado $(\square)$

Department of Electrical Engineering, Institute of Engineering, Polytechnic of Porto, Rua Dr. António Bernardino de Almeida, 431, 4249 - 015 Porto, Portugal e-mail: jtm@isep.ipp.pt

A. M. Lopes

LAETA/INEGI, Faculty of Engineering, University of Porto, Rua Dr. Roberto Frias, 4200 - 465 Porto, Portugal e-mail: aml@fe.up.pt

\section{Introduction}

The age at death is uncertain in nature. With this regard we can quote Thomas Paine "Nothing, they say is more certain than death, and nothing more uncertain than the time of dying". Nevertheless, it is well-known that the risk of death depends on several factors, such as age, sex, genetics, lifestyle, and others. This knowledge comes from the analysis of specific groups of people, often organized per sex and in a country basis. The information is gathered in lifetables, which provide a complete description of mortality in a given population $[17,38,47]$.

Mortality is determinant to the population dynamics and the uncertainty associated either with the time of death, or the risk regarding the duration of life, is crucial in many fields of human activity, such as economy, demography and social sciences [26,31]. In recent years, the topic has received increasing attention, especially due to the trend in population aging in developed countries, showing that people aged 65 or older is increasing faster than all other age groups. Life expectancy statistics give insight about the expected duration of life conditionally on age-specific mortality rates. However, individuals in a population usually do not have idea about the risk relative to their age at death, as well as about the speed at which that risk is resolved as they become older [34]. To tackle these questions, the modeling and forecasting of mortality and the uncertainty in mortality rates have been widely investigated [29,43,49,61]. 
A lifetable is a 2-dimensional array that summarizes the mortality experience of a population and highlights information about longevity [38]. The array lines correspond to age intervals, typically of 1-year width, starting at age 0 and ending at ages greater than 100 years. The array columns stand for several variables computed from population data. The basic information is the probability of death at a given age, which means the probability of a person living until that age to die before his/her next birthday. However, a number of distinct variables and statistics can be derived and included in standard lifetables.

The complementary cumulative distribution function is used in a broad range of applications, including engineering and demography, where it is often denoted by reliability function and survival function, respectively. This mathematical description gives the probability that an "item", or individual, works, or survives, beyond any specified time, or age $[27,63]$. The survival function can be derived from the lifetable and, in the case of human survival, the shape of the curve conveys important information about the population. For example, a more rectangular-like shape means a lower degree of uncertainty about the age at death and, in the limit, a pure rectangular form would correspond to the hypothetical case where all individuals in a population would survive until a specific age and, then, all would die at that same age $[34,60]$. Different measures to quantify death uncertainty have been proposed based on the survival functions. We can mention the Kannisto's coefficient [22], the standard deviation of the age at death $[15,28]$, the Gini index of the length of life [53] and the lifetime entropy [35].

The entropy was developed by Clausius [10] and Boltzmann [8], and later by Shannon [50] and Jaynes [20], in the scope of thermodynamics and information theory, respectively. The entropy has been adopted to characterize complex dynamical systems and used as a measure to quantify uncertainty, diversity and randomness. In the last decades, the original formulation of entropy has been extended by several authors to better adapt to certain systems' characteristics [30].

In demographic sciences, the entropy has been used to quantify the influence of the mortality rate on the expected lifetime $[3,18,19,25]$. A higher entropy means that the expected lifetime is more sensitive to changes in the mortality rate, and vice-versa. The entropy is also regarded as a measure of the "rectangularization" of the survival function. Rectangular-like shapes characterize populations where most people die at older ages and, thus, the volatility of the age at death is small. A full rectangular survival function would translate into zero entropy [37].

The Keyfitz's entropy [25] was proposed to measure the variation of the lifespan. This formulation is a normalized version of the Shannon's expression, and can be interpreted as the "elasticity" of the life expectancy to a change in the mortality. The concept was further explored by Goldman and Lord [19], Vaupel [57], and Vaupel and Canudas Romo [58].

Zhang and Vaupel [62] studied the entropy of the lifetable and showed that there is an age for which averting deaths before (after) that value reduces (increases) life disparity. Therefore, such threshold age separates early and late deaths. If the entropy is less than one, then we have a threshold age that separates early and late deaths. Otherwise, if the entropy is greater than one, then averting deaths at any age increases the disparity. If the entropy has the value one, then preventing deaths at age zero has no effect, but avoiding a death at any age after zero increases the disparity. Aburto et al. [3] derived a formal proof of the threshold age at which age-specific mortality improvements translate into either a reduction or an increase in the lifespan inequality. More recently, Aburto et al. [4] studied the life expectancy and lifespan equality over time. The results demonstrated that the link between both indicators is particularly strong for a life expectancy less than 70 years, and that saving lives at ages below life expectancy is the key to increase the life expectancy and lifespan equality. In this regard, van Raalte and Caswellere [56] studied the response of seven lifespan variation indices with relation to variations in the mortality schedule. The Theil's index and the mean logarithmic deviation [52] are obtained from the entropy of the distribution of the age at death. All indices were derived within the framework of absorbing Markov chains and used to obtain the sensitivity and the elasticity of lifetables with regard to changes in age-specific mortality. Meyer and Ponthiere [34] addressed the quantification of risk regarding the duration of life in the viewpoint of the Shannon's entropy using the logarithm of base 2 . The entropy was interpreted as the expectation of the amount of information (expressed in bits) exhibited by the event of a life of a particular length or, alternatively, of the information disclosed by a death at a given age. Consequently, the lifetime entropy is the informational analogous of the 
life expectancy. In another study, Meyer and Ponthiere [35] investigated the uncertainty about the duration of life, quantified by the Shannon's entropy (measured in bits), due to variations in the age-specific probability of death. They found two threshold ages, namely: (i) a low value, below (above) which a rise in the mortality risk decreases (increases) the lifetime entropy, and (ii) a high value, above which a raise in mortality risk reduces the lifetime entropy.

Reliability engineering addresses the ability of a system, or component, to function under expected conditions for a given period of time. This field deals with the concepts of lifetime uncertainty and risk of failure, among others $[13,16]$. In demography, the counterparts of these concepts are the human lifetime uncertainty and the risk of death, while the systems subjected to failure are people. Therefore, many tools adopted in the area of reliability engineering are identical to those used in the study of human lifetime. The entropy has been widely adopted, specifically the classical formulations and their most common generalizations. Moreover, we can mention the Rényi [42], Tsallis [9], residual [14], cumulative [12] and past [36] entropies, as well as extropies [2], and other closely related indices [21,55].

This paper studies the time at death uncertainty under the perspective of the entropy concept. Data series obtained at the Human Mortality Database for a set of 40 countries are processed by means of several entropy measures. The results unveil patterns that are used to characterize the dynamics of mortality.

The paper includes six sections. Section 2 introduces the concept of entropy and several entropy formulations. Section 3 describes the dataset used in the work. Section 4 analyses the mortality of 40 countries by means of entropy measures. Section 5 compares the different countries using the locus of cumulative residual entropy versus entropy. Finally, Sect. 6 outlines the conclusions.

\section{The concept of entropy}

Let $X$ be a finite discrete random variable with possible values $\left\{x_{1}, x_{2}, \ldots, x_{N}\right\}$ and probability mass function $P=\left\{p_{1}, p_{2}, \ldots, p_{N}\right\}$, where $p_{i}=P\left(X=x_{i}\right)$, $i=1 \ldots, N, \sum_{i} p_{i}=1$ and $p_{i} \geq 0$. The Shannon entropy, $H$, of the random variable $X$ is defined as:

$H=\sum_{i} p_{i} I\left(p_{i}\right)=-\sum_{i} p_{i} \ln p_{i}$.
Expression (1) is the expected value of the information of the event $X=x_{i}$ given by $I\left(p_{i}\right)=-\ln p_{i}$. For the uniform distribution $p_{i}=N^{-1}, N \in \mathbb{N}$, and the Shannon entropy reaches the maximum $H=\ln N$.

The expression (1) can be extended to the continuous case, at the cost of loosing some properties of the discrete formulation [46].

Let us consider a continuous nonnegative random variable $X$ with probability density function $f(x)$. The Shannon differential entropy, $H$, of $X$ is defined as [51]:

$H=-\int_{0}^{\infty} f(x) \ln f(x) \mathrm{d} x$,

and represents a measure of uncertainty for $X$.

The entropy, viewed as a measure of uncertainty in lifetime, attracted attention of several researchers during the last years $[11,23,24,34]$. Let $X$ describe the random lifetime of an "item", and let us denote by $F(x)$ and $\bar{F}(x)$ the cumulative distribution and survival functions of $X$ :

$$
\begin{array}{r}
F(x)=\int_{0}^{x} f(x) \mathrm{d} x, \\
\bar{F}(x)=1-F(x) .
\end{array}
$$

Suppose that the "item" survived up to age $t>0$. We define the Shannon residual entropy of $X$ at time $t, H(t)$, as the entropy of $[X \mid X>t]$, where $[X \mid A]$ stands for probability distribution identical to the one of $X$ conditional on $A$. Therefore, we have [11]:

$$
\begin{aligned}
H(t)= & -\int_{t}^{\infty} \frac{f(x)}{\bar{F}(t)} \ln \frac{f(x)}{\bar{F}(t)} \mathrm{d} x \\
& =1-\frac{1}{\bar{F}(t)} \int_{t}^{\infty} f(x) \ln \lambda(x) \mathrm{d} x,
\end{aligned}
$$

where $\lambda(t)=f(t) / \bar{F}(t)$ is the hazard function, or failure rate, of $X$. By other words, given that the "item" has survived up to time $t$, the residual entropy of $X, H(t)$, reflects the uncertainty about its continuing lifetime.

In certain cases, it is reasonable to presume that uncertainty is not only associated with the future, but is also interconnected to the past. For instance, if the state of an "item" is observed only at certain preassigned time instants and, at the instant $t$, it is found to be not working, or dead, then the uncertainty relies on the past. Therefore, the Shannon past entropy of $X$ at time $t, \bar{H}(t)$, is the differential entropy of the distribution $[X \mid X \leq t]$. The past entropy $\bar{H}(t)$ represents an uncertainty that is the dual of $H(t)$, in the sense that it focuses on the past, and is defined as: 
$\bar{H}(t)=-\int_{0}^{t} \frac{f(x)}{F(t)} \ln \frac{f(x)}{F(t)} \mathrm{d} x$.

We must note that $\bar{H}(t) \in \mathbb{R}$ and that, for $t=0$, expression (4) yields the Shannon differential entropy (2), while expression (5) has no meaning.

Both the residual and past entropies have application in different branches of sciences, namely in reliability engineering [46], computer vision [5], survival analysis [1], image processing [59] and actuarial sciences [48].

A more general measure of uncertainty was proposed by Rao et al. [46], being denoted by Shannon cumulative residual entropy, $\mathcal{E}$, which is obtained when replacing $f(x)$ by $\bar{F}(x)$ in (2), yielding:

$\mathcal{E}=-\int_{0}^{\infty} \bar{F}(x) \ln \bar{F}(x) \mathrm{d} x=\int_{0}^{\infty} \bar{F}(x) \Lambda(x) \mathrm{d} x$,

where $\Lambda(x)=-\ln \bar{F}(x)=\int_{0}^{x} \lambda(t) \mathrm{d} t$ is the so-called cumulative hazard function. The cumulative residual entropy addresses the information content of the distribution of a random variable and thereby is also a measure of uncertainty. This measure is valid in the continuous and discrete domains and can be easily computed from sample data.

Identically, the Shannon cumulative entropy is given by:

$\mathcal{C E}=-\int_{0}^{\infty} F(x) \ln F(x) \mathrm{d} x$.

The dynamic cumulative residual and dynamic cumulative entropies are, in turn, given by [11]:

$$
\begin{aligned}
\mathcal{E}(t) & =-\int_{t}^{\infty} \frac{\bar{F}(x)}{\bar{F}(t)} \ln \frac{\bar{F}(x)}{\bar{F}(t)} \mathrm{d} x \\
& =\int_{t}^{\infty} \frac{\bar{F}(x)}{\bar{F}(t)}[\Lambda(x)-\Lambda(t)] \mathrm{d} x
\end{aligned}
$$

and

$\mathcal{C} \mathcal{E}(t)=-\int_{0}^{t} \frac{F(x)}{F(t)} \ln \frac{F(x)}{F(t)} \mathrm{d} x$.

Several generalizations of expressions (6)-(9), for $n \in \mathbb{N}$, were proposed, such as [55]:

$$
\begin{aligned}
& \mathcal{E}_{n}=\frac{1}{n !} \int_{0}^{\infty} \bar{F}(x)[-\ln \bar{F}(x)]^{n} \mathrm{~d} x, \\
& \mathcal{C} \mathcal{E}_{n}=\frac{1}{n !} \int_{0}^{\infty} F(x)[-\ln F(x)]^{n} \mathrm{~d} x, \\
& \mathcal{E}_{n}(t)=\frac{1}{n !} \int_{t}^{\infty} \frac{\bar{F}(x)}{\bar{F}(t)}\left[-\ln \frac{\bar{F}(x)}{\bar{F}(t)}\right]^{n} \mathrm{~d} x, \\
& \mathcal{C E}_{n}(t)=\int_{0}^{t} \frac{F(x)}{F(t)}\left[-\ln \frac{F(x)}{F(t)}\right]^{n} \mathrm{~d} x .
\end{aligned}
$$

Some properties of $\mathcal{E}$ and $\mathcal{E}(t)$, given by (6) and (8), were discussed by Asadi and Zohrevand [6] and Navarro et al. [39], while the properties of $\mathcal{E}_{n}$ and $\mathcal{E}_{n}(t)$, expressed by (10) and (12), were explored by Navarro and Psarrakos [40], Psarrakos and Navarro [44] and Psarrakos and Toomaj [45].

The generalization of differentiation and entropy has received considerable attention in the last decades. New formulations extended the application of these concepts to highlight characteristics of complex systems $[32,41,54]$. For a recent review on both topics, interested readers can see [30].

Different entropy formulations can have particular interpretations in the scope of a given application [7]. In the present case, we shall adopt a more abstract point of view and consider them as alternative information measures for capturing distinct properties of the system under study. Indeed, an eclectic assessment of the dataset seems a better strategy to avoid a priori assumptions that may overlook relevant information embedded in the dataset.

\section{Brief description of the dataset}

Data for 40 countries (Table 1 in the Appendix) were collected at the Human Mortality Database website on February 2, 2021 (https://www.mortality.org/). The Human Mortality Database, held by the University of California, Berkeley, and Max Planck Institute for Demographic Research, contains original information of death rates and lifetables for national populations. The lifetables are available for men, women and both genders, in an annual basis. The age intervals have 1year width, starting at the age $t=0$ (i.e., birth) and ending at age $t=110$, meaning that the last interval includes ages $t>110$. The probabilities of death, $q(t)$, and other indicators of mortality and longevity are given at each age $t$. The data covers different periods of time for each country. The longest and shortest records are those collected for Sweden and the Republic of Korea comprising 269 and 16 years, respectively.

From the lifetables we derive the survival function, $\bar{F}(t)$, as:

$\bar{F}(t+1)=[1-q(t)] \cdot \bar{F}(t), \quad t=1,2, \ldots, 109$,

with $\bar{F}(0)=1$. 
Fig. 1 The probability density and survival functions, $f$ and $\bar{F}$, versus calendar time and age, $\tau$ and $t$ (expressed in years), for the whole population (male plus female) lifetables of France and Norway: a $f$ for France; $\mathbf{b} \bar{F}$ for France; c $f$ for Norway; (d) $\bar{F}$ for Norway. The probability density function, $f$, is in $\log$ units. The colorbar represents $f(\tau, t)$ in a logarithmic scale
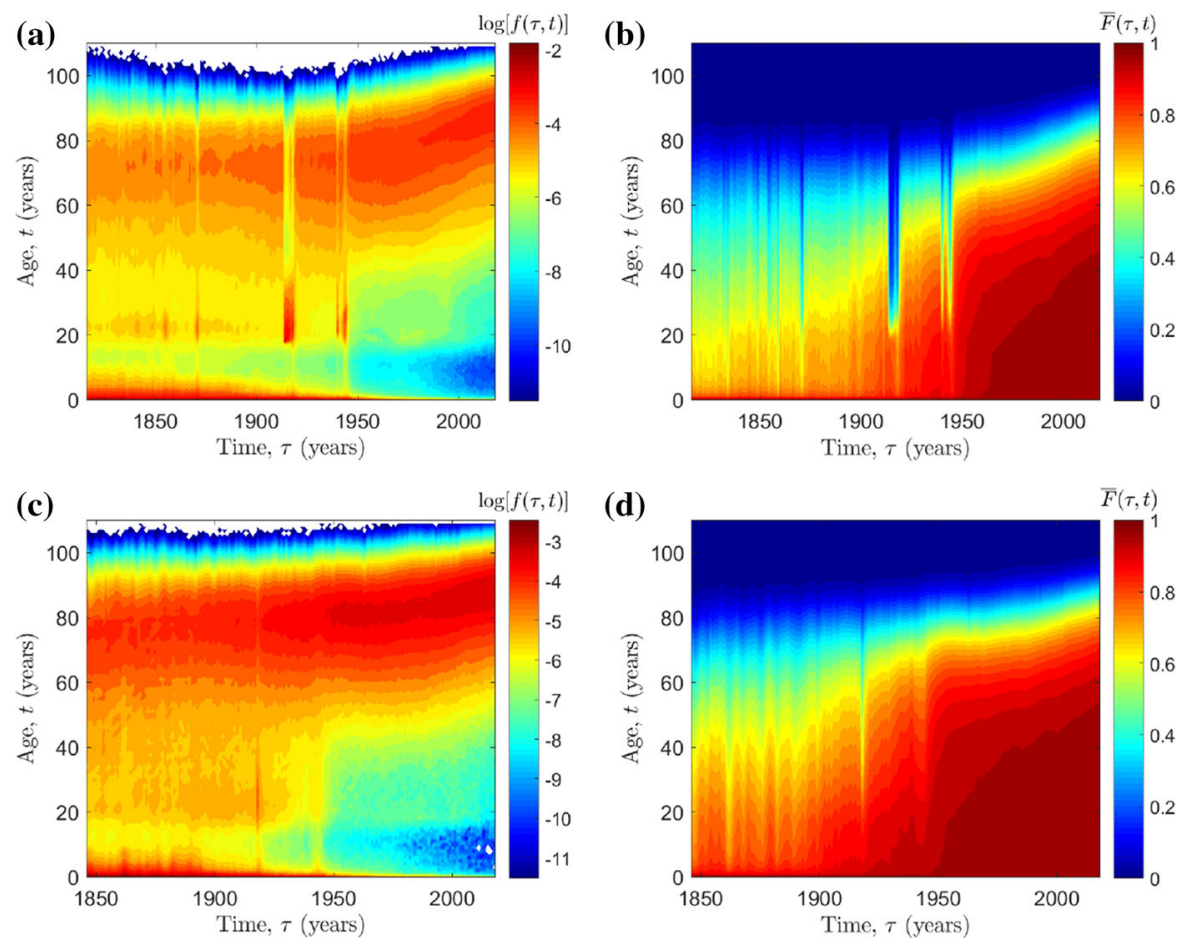

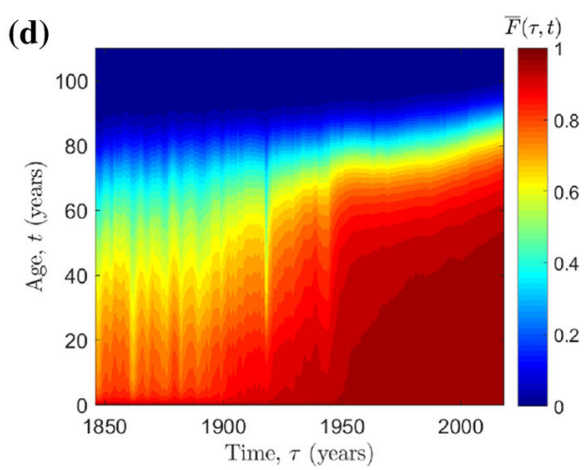

The probability density function, $f(t)$, is computed by:

$f(t)=\frac{\mathrm{d}}{\mathrm{d} t} F(t)$.

Figure 1 depicts the functions $f$ and $\bar{F}$ versus calendar time and age, $\tau$ and $t$ (expressed in years), for the whole population (male plus female) lifetables of France and Norway.

We verify that the probability of death is high for newborn and for $t \in[65,90]$ years old people. Above the age $t \approx 90$ years the probability of death drops very fast towards zero. Moreover, we observe that for children the mortality has been decreasing in calendar time, while for the group $t \in[65,90]$ years the age of maximum probability of death has been increasing. The effect of certain extreme events, as the two World Wars (WWI and WWII) are well visible in the plots. The other countries have $f$ and $\bar{F}$ patterns of the same type.

\section{Human lifetime entropy}

This section analyses the lifetime entropy along calendar time and age, $\tau$ and $t$, using data of period lifetables. For a given year, $\tau$, the lifetime entropy quantifies the amount of risk, or uncertainty, about the age at death that is unresolved at age $t$. Therefore, for the particular case of $t=0$, we obtain the lifetime entropy at birth, which represents the risk of a complete lifetime.

Figure 2 depicts the Shannon and cumulative residual entropies versus time and age, $H(\tau, t)$ and $\mathcal{E}(\tau, t)$, for the France and Norway complete populations (i.e., males plus females). By other words, for each year, $\tau$, the entropies are calculated for the variable $t$ (i.e., the age) by means of expressions (4) and (8). We verify that the lifetime entropy is higher at younger ages and reduces progressively as age increases. This means that the duration of life is progressively resolved when individuals become older. In fact, at younger ages numerous factors can affect the duration of life and make it more unpredictable, which translates into larger entropy. On the contrary, at older ages, the number of possible scenarios that determine the duration of life becomes more limited, and the lifetime entropy is reduced. In this perspective, the lifetime entropy at successive ages pictures how the amount of risk regarding the duration of life varies with age and how it evolves when individuals become older [35]. For other countries the results are of the same type. 
Fig. 2 The values of Shannon and dynamic cumulative residual entropies, $H(\tau, t)$ and $\mathcal{E}(\tau, t)$, versus calendar time and age, $\tau$ and $t$, for the populations of: a France; $\mathbf{b}$ Norway
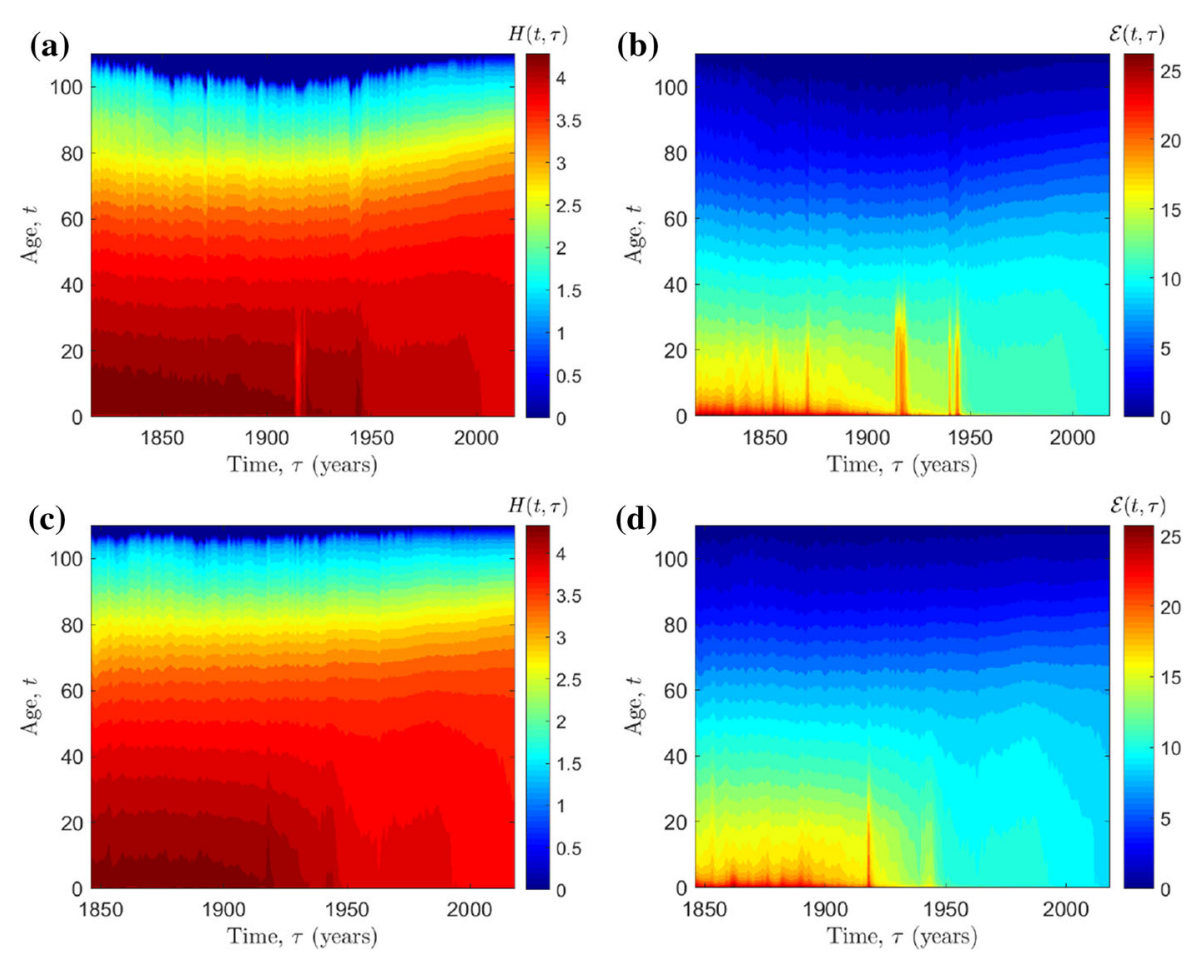

Comparing these plots with those depicted in Fig. 1, we note that we reduce slightly the visibility of the WWI and WWII periods, but, on the other hand, we obtain a more uniform variation with the age $t$.

It should also be mentioned that other entropy formulations were experimented and led to results of the same type as those presented. Naturally, the analysis can also be performed for groups, namely organized by gender or by age, instead for the whole country population.

\subsection{Residual lifetime entropy}

We compare countries based on the uncertainty about remaining lifetimes. We consider $H(\tau, t)$ and $\mathcal{E}(\tau, t)$ for the years $\tau=\{1958,1978,1998,2018\}$ and ages $t=\{0, \ldots, 110\}$. The set covers a wide period of time. We must note that lifetables are not available for some countries during all years and, therefore, the lines exhibit different starting and ending dates.

Figures 3 and 4 depict the Shannon and dynamic cumulative residual entropies, $H(\tau, t)$ and $\mathcal{E}(\tau, t)$, and reveal that the countries exhibit identical entropy patterns. Two notable points emerge, where the slope of the curves changes considerable and, simultaneously, the entropy values approach to each other. The first point corresponds approximately to age threshold value $t_{h 1}=52$ years in year $\tau=1958$ and increases until $t_{h 1}=65$ in $\tau=2018$. The second point corresponds to age $t_{h 2}=105$ years and practically does not change in time.

The variation of the threshold values $t_{h 1}$ and $t_{h 2}$ is also noticeable when analyzing a given country. For instance, Fig. 5 represents $t_{h 1}$ and $t_{h 2}$ for France and Norway. We verify that, for France, $t_{h 1}$ increases slowly from $t=62$ at $\tau=1816$ up to $t=67$ at $\tau=1960$. Then, $t_{h 1}$ increases faster, reaching approximately $t=$ 75 at $\tau=2018$. The threshold $t_{h 2}$ is almost constant. For Norway, $t_{h 1}$ increases from $t=52$ at $\tau=1816$ up to $t=60$ at $\tau=1890$. For $\tau \in[1890,1975], t_{h 1}$ increases, reaching approximately the value $t=65$ at $\tau=2017$. The threshold $t_{h 2}$ is somewhat noisy, but maintains its constant trend over time. We can conjecture that $t_{h 1}$ reflects public health policies that led to an higher expectancy of life, while $t_{h 2}$ points to some intrinsic biological limit, but we must highlight that this is merely an interpretation based on the numbers. 
(a)

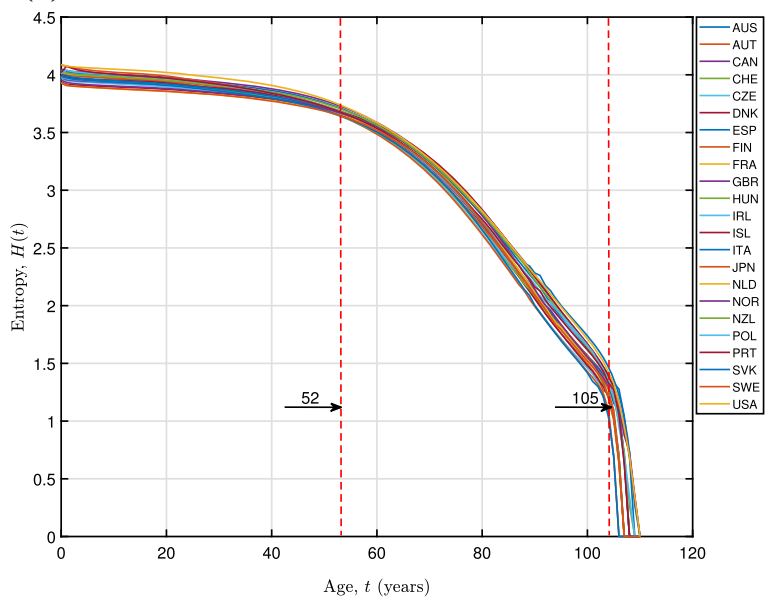

(c)

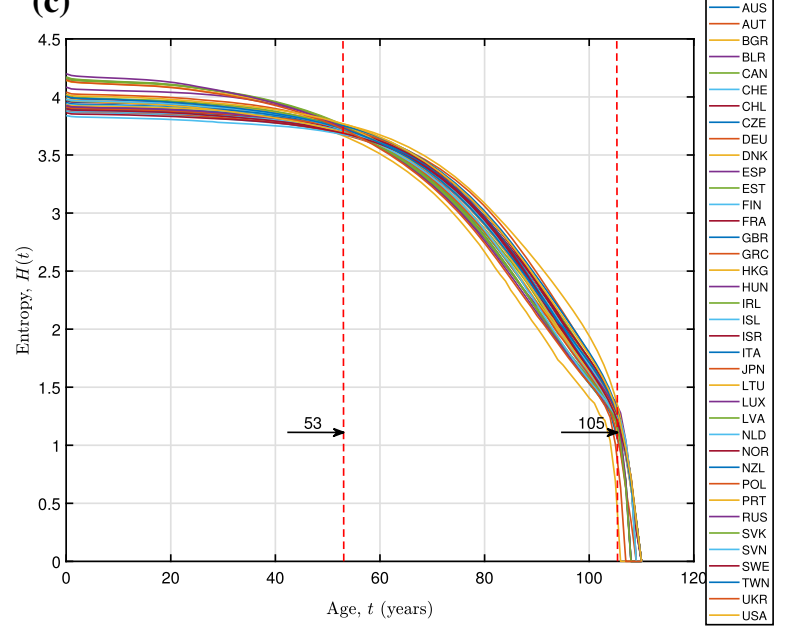

(b)

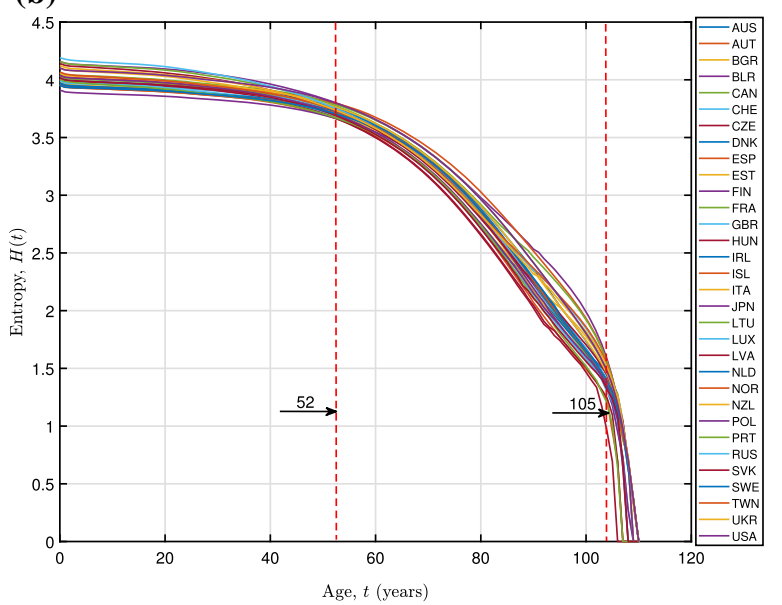

(d)

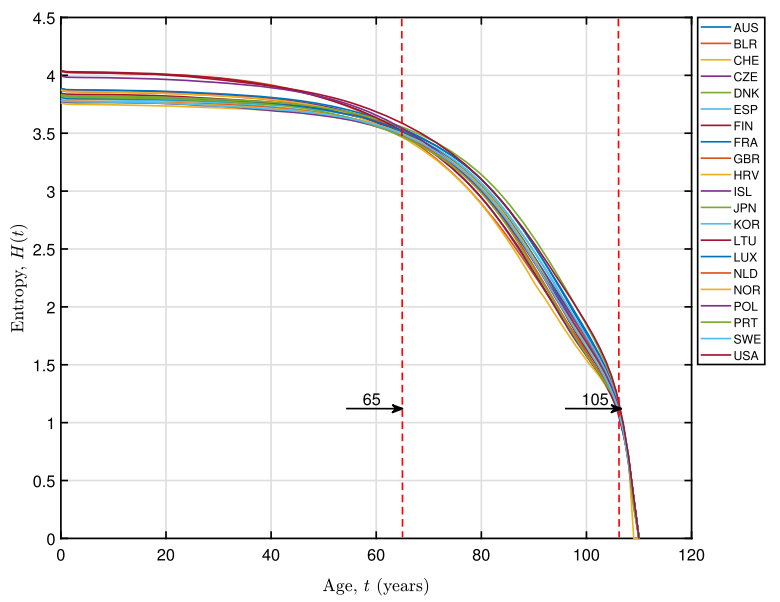

Fig. 3 The Shannon entropy $H(\tau, t)$ of various countries for 4 years and all ages $t=\{0, \ldots, 110\}$ : $\mathbf{a} \tau=1958$; b $\tau=1978$; c $\tau=1998 ; \mathbf{d} \tau=2018$

\subsection{Period lifetime entropy}

We now compare all countries based on the evolution of their lifetime entropy versus time $\tau$. As such, we compute $H(\tau, t)$ and $\mathcal{E}(\tau, t)$, for all years, $\tau$, and a set of six ages $t=\{0,25,45,60,75,85\}$.

Figures 6 and 7 depict the entropies $H(\tau, t)$ and $\mathcal{E}(\tau, t)$, respectively, for the 40 countries listed in Table 1 when considering the whole country populations.

We verify that $H$ is quite noisy until around year $\tau=1880$, probably because the information had to be tackled manually, with strong limitations in collecting and communicating data, and no computational resources available to construct and maintain accurate datasets. For $t=0$, we can see that $H$ increases in time until approximately year $\tau=1914$, corresponding to the beginning of WWI, while we observe a convergence of its values for all countries. The effect of the WWI $(1914-1918)$ is clear, translating into a perturbation characterized by a local maximum at year $\tau=1918$, for most countries, and a minimum for France, Italy and Spain. Between the two World Wars, the entropy decreases slowly, while the convergence between its behavior for all countries continues. During the WWII $(1939$ - 1945) the entropy increases in time. In the decade post WWII, the values fall down quickly and the country entropies reveal maximum convergence. 
(a)

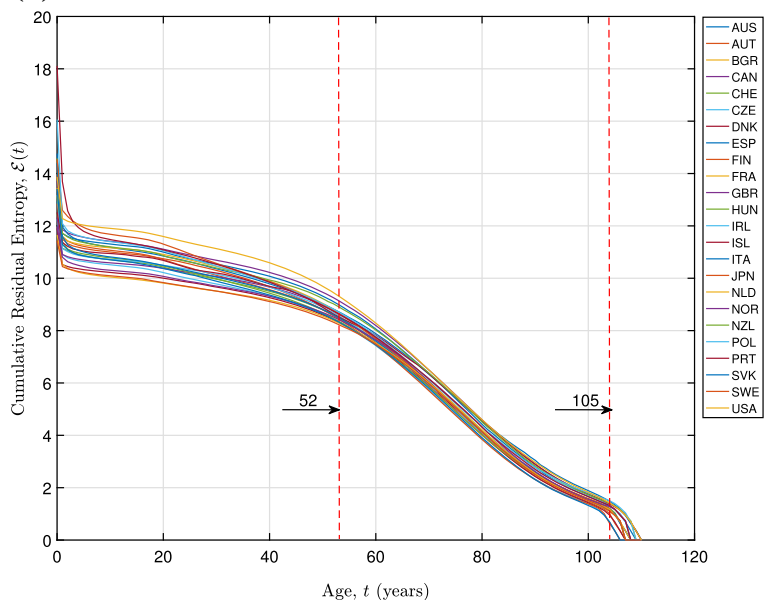

(c)

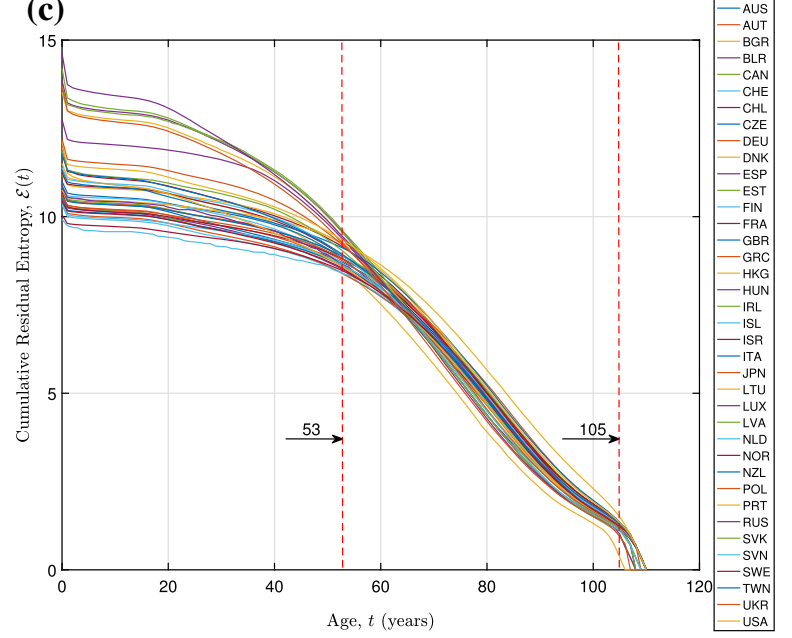

(b)

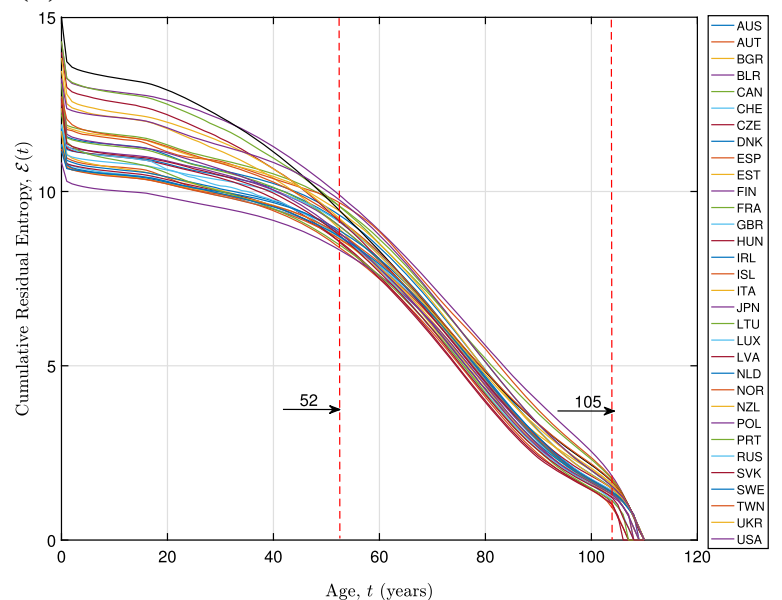

(d)

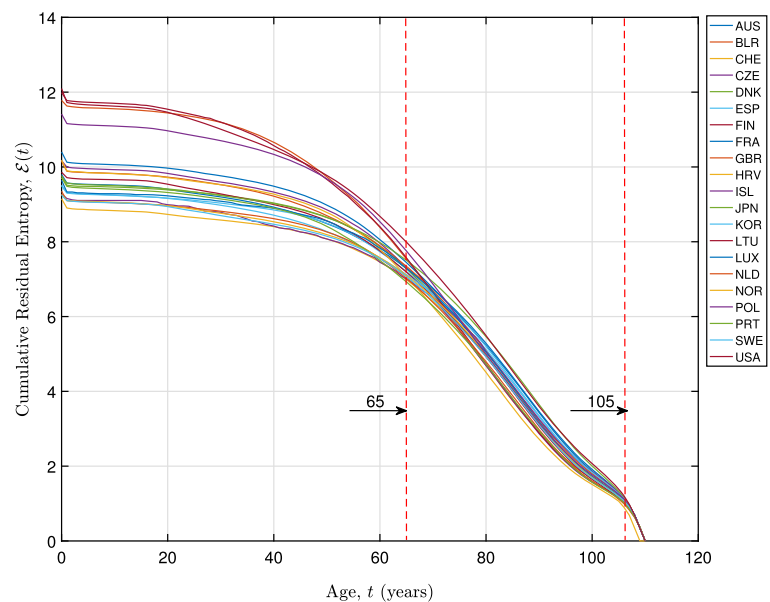

Fig. 4 The dynamic cumulative residual entropy $\mathcal{E}(\tau, t)$ of various countries for 4 years and all ages $t=\{0, \ldots, 110\}: \mathbf{a} \tau=1958 ; \mathbf{b}$ $\tau=1978 ; \mathbf{c} \tau=1998 ; \mathbf{d} \tau=2018$

Fig. 5 The variation of the threshold values $t_{h 1}$ and $t_{h 2}$ for: a France; b Norway (a)

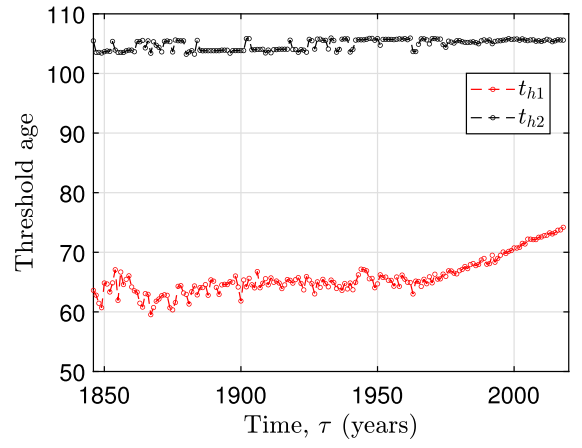

(b)

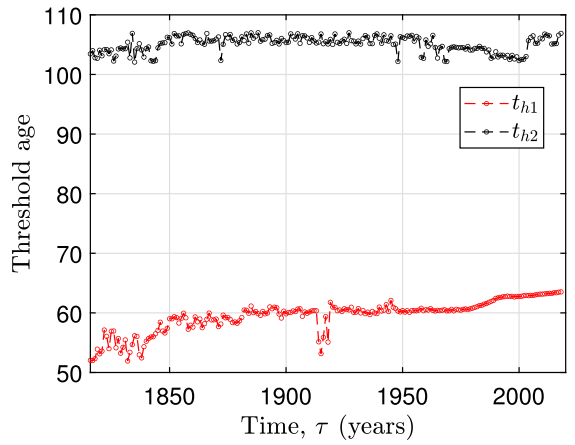


(a)

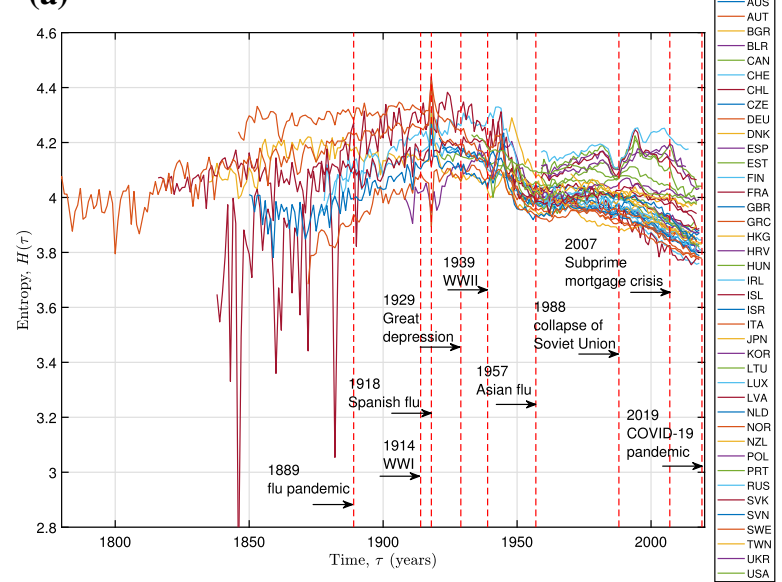

(c)

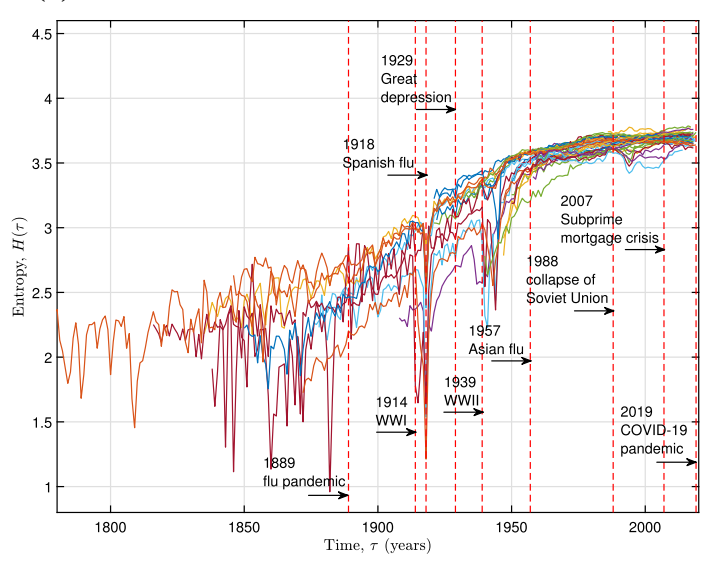

(e)

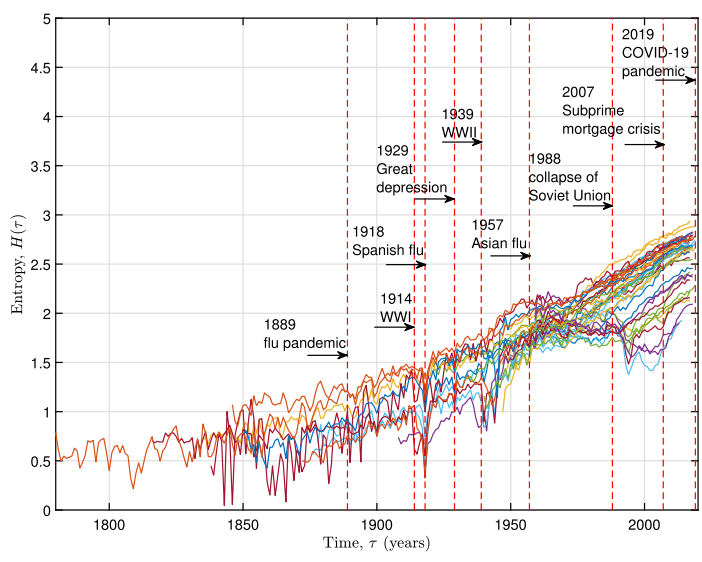

(b)

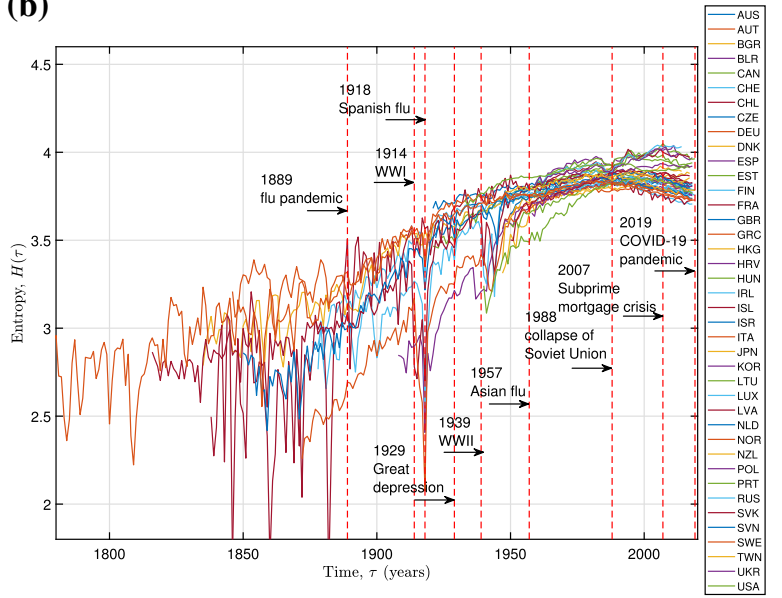

(d)

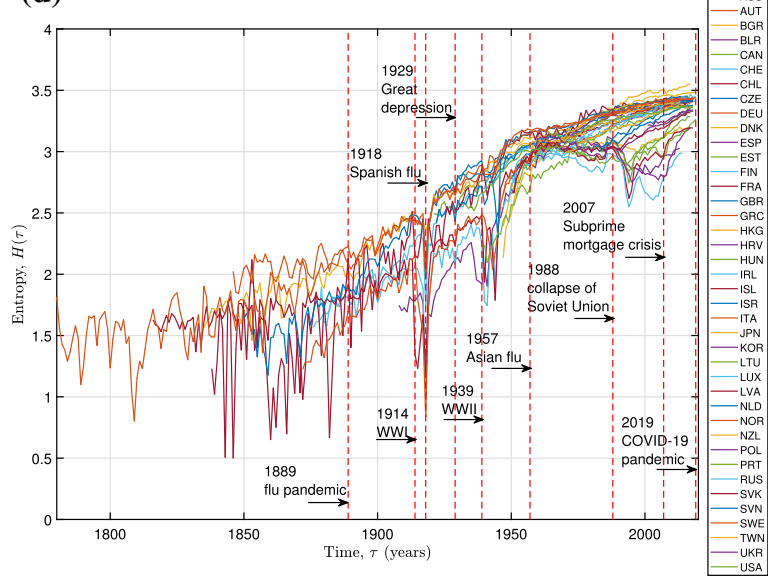

(f)

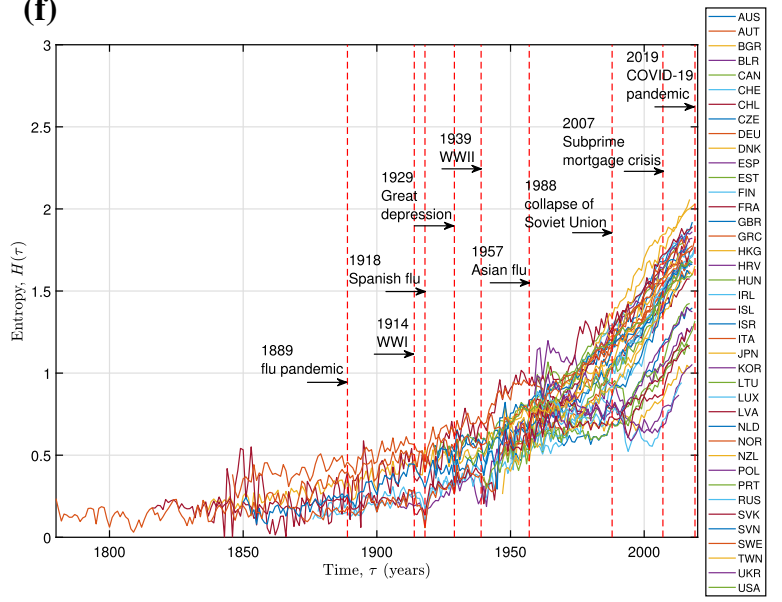

Fig. 6 The Shannon entropy $H(\tau, t)$ of various countries for $t \in[1751,2019]$ and the ages: $\mathbf{a} t=0 ; \mathbf{b} t=25$; $t=45$; $\mathbf{d} t=60$; $\mathbf{e}$ $t=75 ; \mathbf{f} t=85$ 
(a)

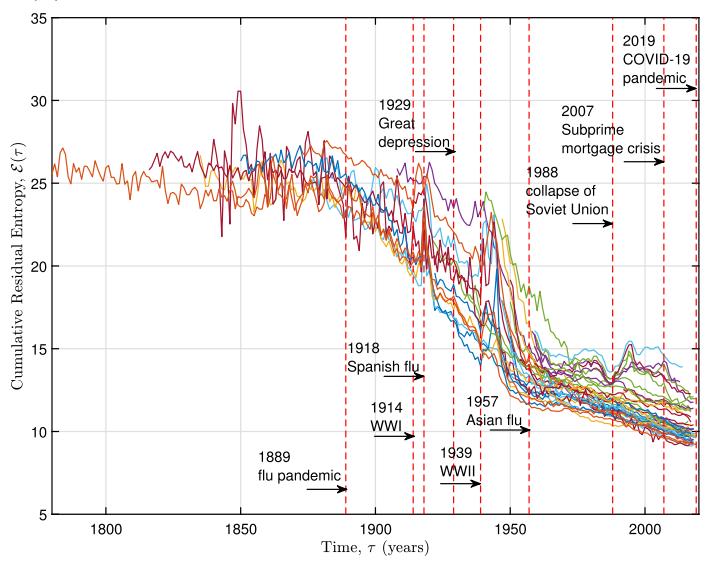

(c)

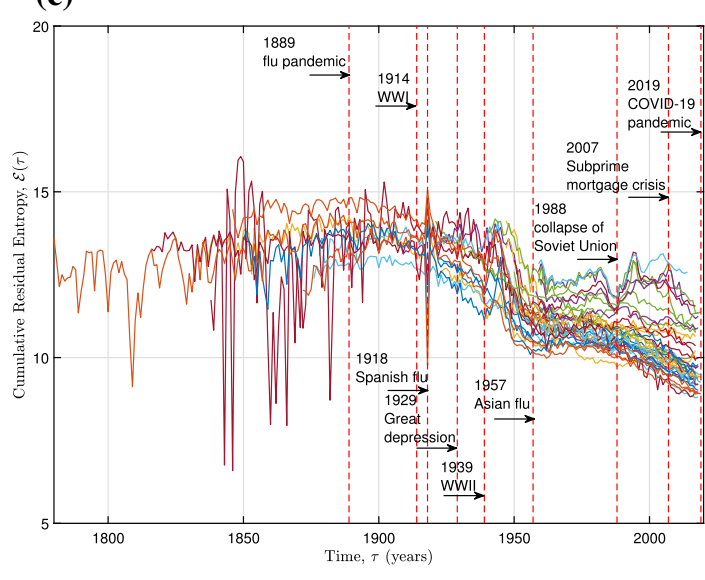

(e)

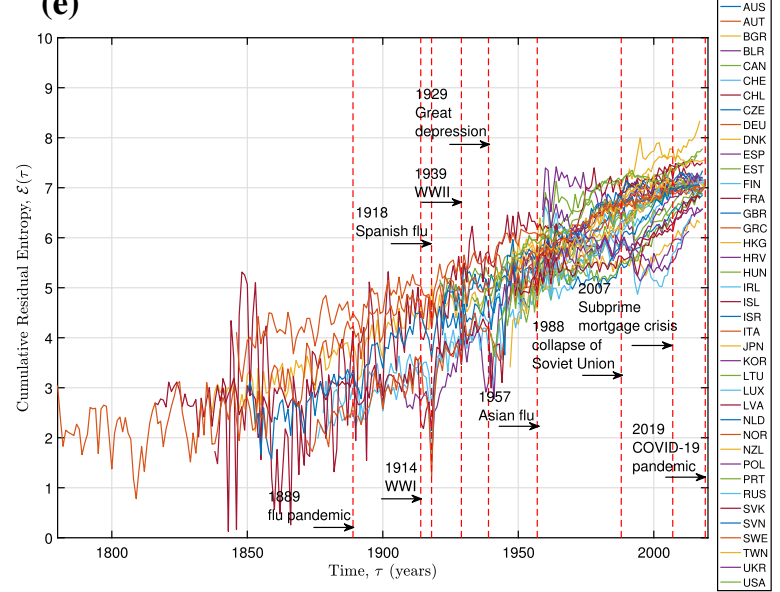

(b)

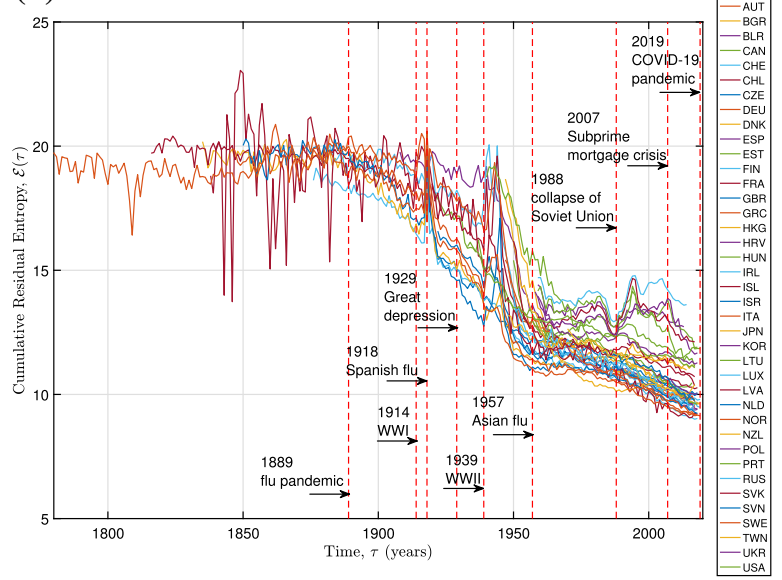

(d)

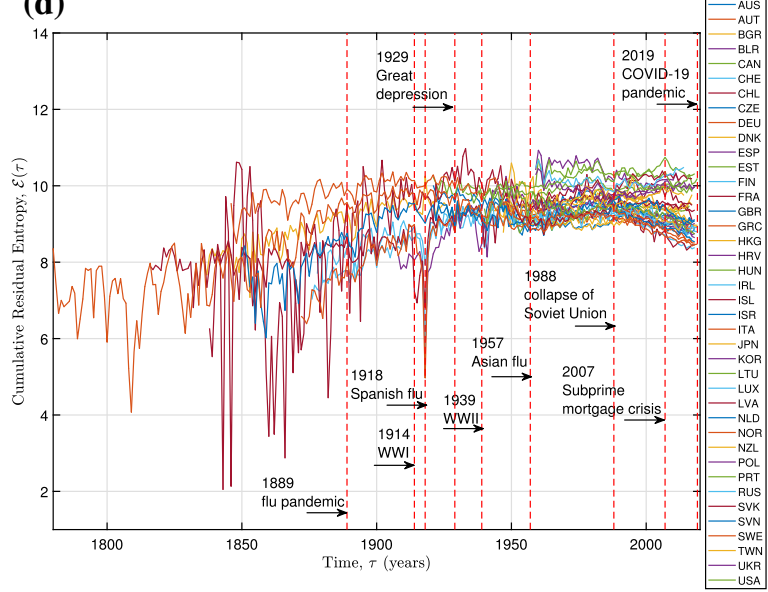

(f)

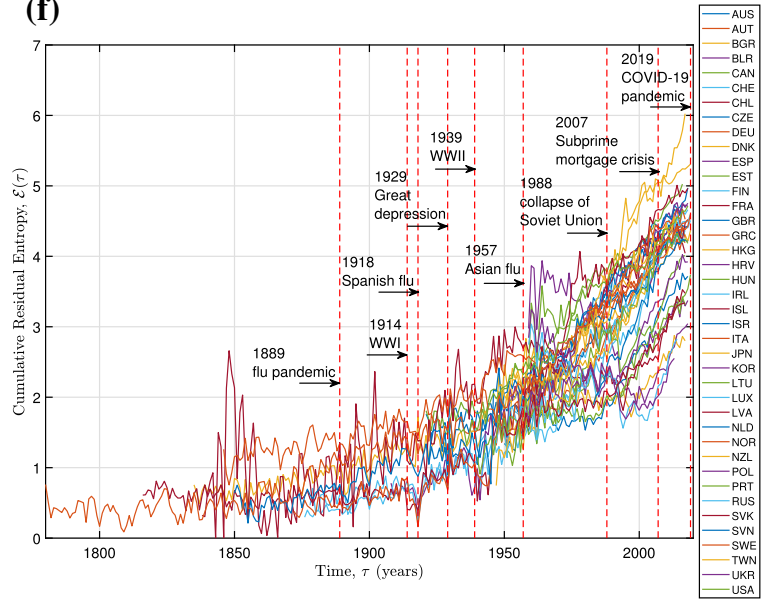

Fig. 7 The dynamic cumulative residual entropy $\mathcal{E}(\tau, t)$ of various countries for $t \in[1751,2019]$ and the ages: a $t=0 ; \mathbf{b} t=25$; $\mathbf{c}$ $t=45 ; \mathbf{d} t=60 ; \mathbf{e} t=75 ; \mathbf{f} t=85$ 
For most countries, this effect is followed by a relative stable period that goes from year $\tau=1957$, the beginning of the "Asian flu" pandemic, until year $\tau=1988$, the beginning of the collapse of the Soviet Union. Then, from $\tau=1994$ onward, the entropy diminishes moderately in time. Simultaneously, for a group of Eastern Europe countries, the entropy firstly increases slowly, has clear local minimum and maximum around years $\tau=1988$ and $\tau=1994$, respectively, and diminishes moderately from $\tau=1994$ onward. Starting around year $\tau=1957$ and becoming more evident from year $\tau=1988$ onward, the 40 countries separate in three clusters: $\mathcal{C}_{1}=\{$ BLR, EST, LTU, LVA, RUS, UKR $\}$, $\mathcal{C}_{2}=\{$ BGR, CHL, HUN, POL, SVK, TWN, USA $\}$ and $\mathcal{C}_{3}=\{$ AUS, AUT, CAN, CHE, CZE, DEU, DNK, ESP, FIN, FRA, GBR, GRC, HKG, HRV, IRL, ISL, ISR, ITA, JPN, KOR, LUX, NLD, NOR, NZL, PRT, SVN, SWE\}. The effect of other extreme events in the behavior of $H$ is not visible.

For age $t=25$ the entropy increases until approximately year $\tau=2007$ and then it inverts the tendency. There is a trend to convergence between values for all countries. This behavior is even more evident at age $t=45$ years. For age $t=60$ the general entropy pattern changes and, for $t=75$ and $t=85$, the clusters identified at $t=0$ show up again.

In what concerns the plot of the dynamic cumulative residual entropy $\mathcal{E}$ versus time (Fig. 7), we verify the existence of three main periods for the ages $t=\{0,25,45\}$. The first period lasts until approximately year $\tau=1880$, where $\mathcal{E}$ is roughly constant, the second period is $\tau \in[1880,1957]$ with $\mathcal{E}$ diminishing considerably, and the third goes from $\tau=1957$ onward, characterized by moderate decreasing values of $\mathcal{E}$. Moreover, it can be seen that (i) $\mathcal{E}$ is less noisy that $H$, (ii) the period $\tau \in[1880,1957]$ exhibits large differences among countries, with the effect of the two World Wars clearly visible through the peak values, and (iii) in the period from $\tau=1957$ onward the clusters identified for $H$ are also present. For the age $t=60$ the pattern changes and, for $t=75$ and $t=85$, the entropy increases over time. Therefore, $\mathcal{E}$ characterizes long-period trends better than $H$, while signaling also the local behavior of lifetime. We observe also that $H$ and $\mathcal{E}$ exhibit some correlation, but they highlight different characteristics of the lifetime.

\section{The locus of dynamic cumulative residual entropy versus Shannon entropy}

We consider here that $H(\tau)$ and $\mathcal{E}(\tau)$, are the state variables of a dynamical system describing human lifetime.

Figure 8 shows the locus of $\mathcal{E}(\tau)$ versus $H(\tau)$ for the 40 countries at ages $t=\{0,25,45,60,75,85\}$. We have a similar behavior for all countries and ages. However, we verify that there is a change in the patterns over time, as already noticed in Figs. 6 and 7. In fact, we note a modification of the shape of the locus for ages between $t=60$ and $t=75$, which is consistent with the previous observations for $t_{h 1}$. In what concerns viewing the influence of $t_{h 2}$, we have difficulties due to the lack of data. In fact, the loci for high values of the age are simply a collection of points with a limited scatter along a simple straight line. This means, somehow, that at such ages and at present state of public health, we have presently an evolution with almost a deterministic nature.

Loosely speaking, we can say that with regard with the topic of this paper each country has it own, and distinct, calendar time. Nonetheless, the differences between countries have reduced significantly along years. Therefore, we can say that some close "synchronization" between countries will occur in the years to come. At the time of writing this paper we have the COVID-19 pandemic [33], but no data is yet available. It will be an interesting future study to assess its effect upon the results discussed here.

\section{Conclusion}

This paper analyzed death uncertainty through the concept of entropy. A set of 40 countries was studied based on different entropy measures. The entropy patterns were used to characterize the dynamics of mortality. Contrary to some results reported in the literature, in the last decades country lifetime entropy is not converging to a single point. The convergence to several distinct values started approximately in 1957. Since then, three clusters emerged, their relative differences firstly increased and, then, stabilized. The evolution of the entropy in time and the locus of the dynamic cumulative residual entropy versus Shannon entropy led to relevant results. The locus, in particular, is more advan- 
(a)

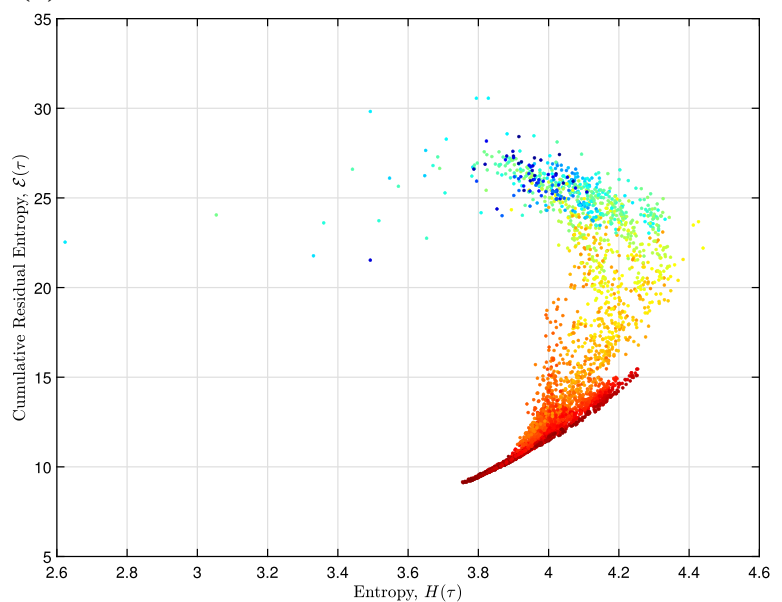

(c)

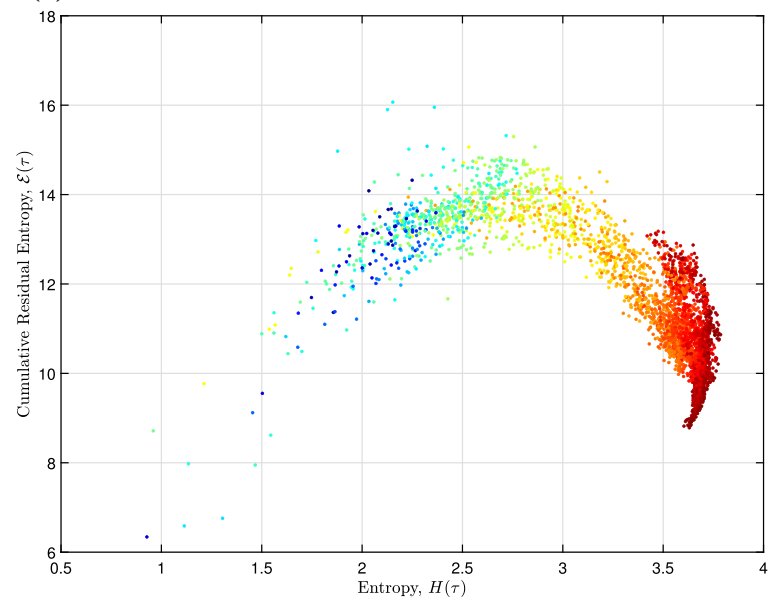

(e)

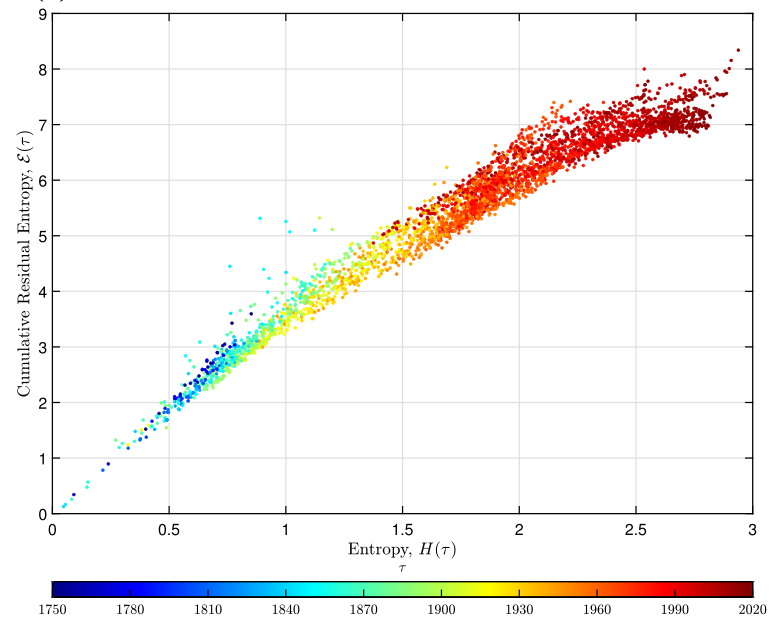

(b)

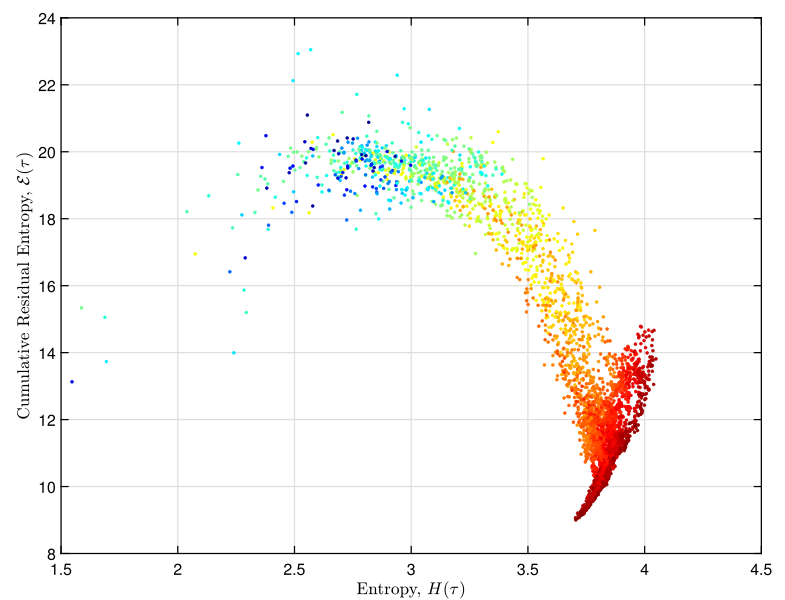

(d)

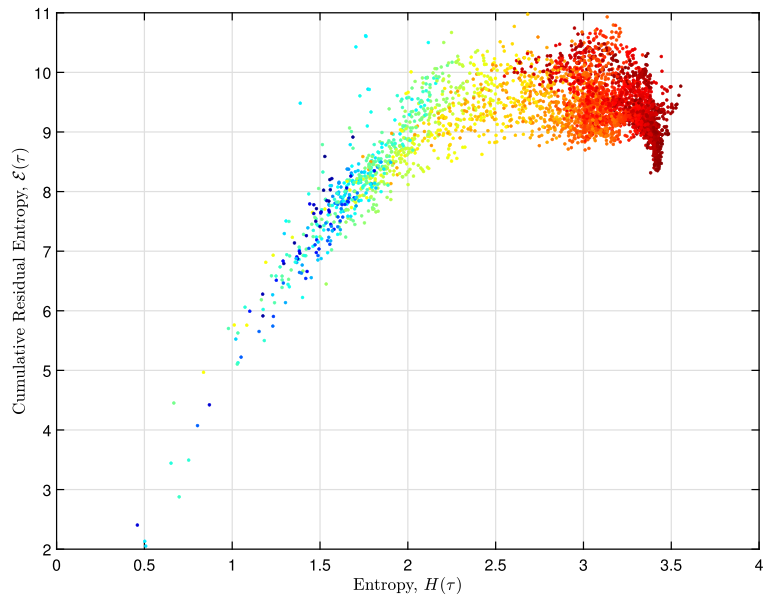

(f)

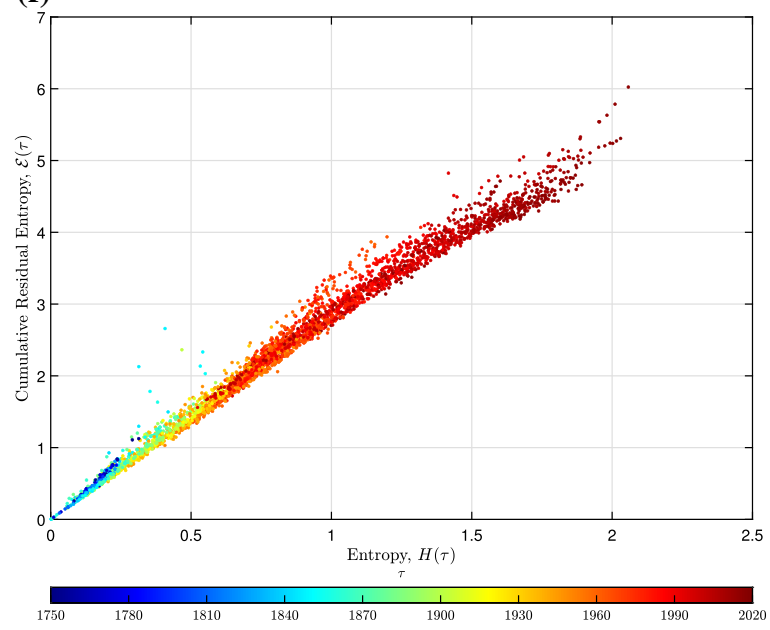

Fig. 8 The locus of $\mathcal{E}(\tau)$ versus $H(\tau)$ for the set of 40 countries and the ages: a $t=0 ; \mathbf{b} t=25 ; \mathbf{c} t=45 ; \mathbf{d} t=60$; e $t=75 ; \mathbf{f}$ $t=85$. Each point corresponds to one year and the arrow of time flows from left to right 
tageous since brings into evidence long range patterns

\section{Appendix}

embedded in the data.

\section{Declarations}

Conflict of interest The authors declare that they have no conflict of interest

Table 1 List of countries with dataset time ranges

\begin{tabular}{|c|c|c|c|c|c|c|c|c|c|}
\hline Number & Name & Acronym & Start year & End year & Number & Name & Acronym & Start year & End year \\
\hline 1 & Australia & AUS & 1921 & 2018 & 21 & Japan & JPN & 1947 & 2019 \\
\hline 2 & Austria & AUT & 1947 & 2017 & 22 & Latvia & LVA & 1959 & 2017 \\
\hline 3 & Belarus & BLR & 1959 & 2018 & 23 & Lithuania & LTU & 1959 & 2019 \\
\hline 4 & Bulgaria & BGR & 1947 & 2017 & 24 & Luxembourg & LUX & 1960 & 2019 \\
\hline 5 & Canada & CAN & 1921 & 2016 & 25 & Netherlands & NLD & 1850 & 2018 \\
\hline 6 & Chile & CHL & 1992 & 2017 & 26 & New Zealand & NZL & 1948 & 2013 \\
\hline 7 & Croatia & HRV & 2001 & 2018 & 27 & Norway & NOR & 1846 & 2018 \\
\hline 8 & Czechia & CZE & 1950 & 2018 & 28 & Poland & POL & 1958 & 2018 \\
\hline 9 & Denmark & DNK & 1835 & 2019 & 29 & Portugal & PRT & 1940 & 2018 \\
\hline 10 & Estonia & EST & 1959 & 2017 & 30 & Republic of Korea & KOR & 2003 & 2018 \\
\hline 11 & Finland & FIN & 1878 & 2019 & 31 & Russia & RUS & 1959 & 2014 \\
\hline 12 & France & FRA & 1816 & 2018 & 32 & Slovakia & SVK & 1950 & 2017 \\
\hline 13 & Germany & DEU & 1990 & 2017 & 33 & Slovenia & SVN & 1983 & 2017 \\
\hline 14 & Greece & GRC & 1981 & 2017 & 34 & Spain & ESP & 1908 & 2018 \\
\hline 15 & Hong Kong & HKG & 1986 & 2017 & 35 & Sweden & SWE & 1751 & 2019 \\
\hline 16 & Hungary & HUN & 1950 & 2017 & 36 & Switzerland & $\mathrm{CHE}$ & 1876 & 2018 \\
\hline 17 & Iceland & ISL & 1838 & 2018 & 37 & Taiwan & TWN & 1970 & 2014 \\
\hline 18 & Ireland & IRL & 1950 & 2017 & 38 & U.K. & GBR & 1922 & 2018 \\
\hline 19 & Israel & ISR & 1983 & 2016 & 39 & U.S.A. & USA & 1933 & 2018 \\
\hline 20 & Italy & ITA & 1872 & 2017 & 40 & Ukraine & UKR & 1959 & 2013 \\
\hline
\end{tabular}




\section{References}

1. Abbasnejad, M., Arghami, N.R., Morgenthaler, S., Borzadaran, G.M.: On the dynamic survival entropy. Statist. Probab. Lett. 80(23-24), 1962-1971 (2010)

2. Abdul Sathar, E., Nair R, D.: On dynamic survival extropy. Commun. Statis-Theory Meth. pp. 1-19 (2019)

3. Aburto, J.M., Alvarez, J.A., Villavicencio, F., Vaupel, J.W.: The threshold age of the lifetable entropy. Demograp. Res. 41, 83-102 (2019)

4. Aburto, J.M., Villavicencio, F., Basellini, U., Kjærgaard, S., Vaupel, J.W.: Dynamics of life expectancy and life span equality. Proc. Nat. Acad. Sci. 117(10), 5250-5259 (2020)

5. Al-Shabi, M.: A novel edge detection algorithm based on cumulative residual entropy. Applied Mathematics 14(2), 319-326 (2020)

6. Asadi, M., Zohrevand, Y.: On the dynamic cumulative residual entropy. J. Statist. Plan. Inf. 137(6), 1931-1941 (2007)

7. Ben-Naim, A.: Entropy: The truth, the whole truth and nothing but the truth. World Scientific, (2017)

8. Boltzmann, L.: Vorlesungen über die Principe der Mechanik, vol. 1. JA Barth (1897)

9. Calì, C., Longobardi, M., Ahmadi, J.: Some properties of cumulative Tsallis entropy. Phys. A 486, 1012-1021 (2017)

10. Clausius, R.: The mechanical theory of heat: with its applications to the steam-engine and to the physical properties of bodies. J. van Voorst (1867)

11. Di Crescenzo, A., Longobardi, M.: Entropy-based measure of uncertainty in past lifetime distributions. J. Appl. Prob. 434-440 (2002)

12. Di Crescenzo, A., Longobardi, M.: On cumulative entropies. J. Statist. Plan. Inf. 139(12), 4072-4087 (2009)

13. Du, Y.M., Ma, Y.H., Wei, Y.F., Guan, X., Sun, C.: Maximum entropy approach to reliability. Phys. Rev. E 101(1), 012106 (2020)

14. Ebrahimi, N.: How to measure uncertainty in the residual life time distribution. Sankhyā: The Indian J. Statist. Series A, pp. 48-56 (1996)

15. Edwards, R.D., Tuljapurkar, S.: Inequality in life spans and a new perspective on mortality convergence across industrialized countries. Populat. Develop. Rev. 31(4), 645-674 (2005)

16. Elsayed, E.A.: Reliability engineering. Wiley (2020)

17. Fergany, N.: On the human survivorship function and life table construction. Demography 8(3), 331-334 (1971)

18. Fernandez, O.E., Beltrán-Sánchez, H.: The entropy of the life table: A reappraisal. Theor. Popul. Biol. 104, 26-45 (2015)

19. Goldman, N., Lord, G.: A new look at entropy and the life table. Demography 23(2), 275-282 (1986)

20. Jaynes, E.T.: Information theory and statistical mechanics. Phys. Rev. 106(4), 620 (1957)

21. Kamari, O., Buono, F.: On extropy of past lifetime distribution. Ricerche di Matematica, pp. 1-11 (2020)

22. Kannisto, V.: Measuring the compression of mortality. Demograph. Res. 3, (2000)

23. Kapodistria, S., Psarrakos, G.: Some extensions of the residual lifetime and its connection to the cumulative residual entropy. Probability in the Engineering and Informational Sciences 26(1), 129 (2012)
24. Kayal, S., Moharana, R.: On weighted cumulative residual entropy. J. Statist. Manag. Syst 20(2), 153-173 (2017)

25. Keyfitz, N.: Applied mathematical demography. Springer, (2005)

26. Kleiber, C., Kotz, S.: Statistical size distributions in economics and actuarial sciences, vol. 470. Wiley (2003)

27. Kleinbum, D., Klein, M.: Survival analysis: a self-learning text. Springer (2012)

28. Lan Karen Cheung, S., Robine, J.M.: Increase in common longevity and the compression of mortality: the case of Japan. Populat. Stud. 61(1), 85-97 (2007)

29. Li, S.H., Chan, W.S.: The lee-carter model for forecasting mortality, revisited. North Am. Act. J. 11(1), 68-89 (2007)

30. Lopes, A.M., Machado, J.A.T.: A review of fractional order entropies. Entropy 22(12), 1374 (2020)

31. Macdonald, A.S., Richards, S.J., Currie, I.D.: Modelling mortality with actuarial applications. Cambridge University Press (2018)

32. Machado, J.T., Lopes, A.M.: The persistence of memory. Nonlinear Dyn. 79(1), 63-82 (2015)

33. Machado, J.T., Lopes, A.M.: Rare and extreme events: the case of COVID-19 pandemic. Nonlinear Dyn. 100, 29532972 (2020)

34. Meyer, P., Ponthière, G.: Human lifetime entropy in a historical perspective (1750-2014). Cliometrica 14(1), 129-167 (2020)

35. Meyer, P., Ponthiere, G.: Threshold ages for the relation between lifetime entropy and mortality risk. Mathem. Soc. Sci. 108, 1-7 (2020)

36. Minimol, S.: On generalized dynamic cumulative past entropy measure. Commun. Statist. Theory Meth. 46(6), 2816-2822 (2017)

37. Nagnur, D.: Rectangularization of the survival curve and entropy: The Canadian experience, 1921-1981. Canadian Studies in Population [ARCHIVES], pp. 83-102 (1986)

38. Namboodiri, K., Suchindran, C.M.: Life table techniques and their applications. Academic Press, (2013)

39. Navarro, J., del Aguila, Y., Asadi, M.: Some new results on the cumulative residual entropy. J. Statist. Plan. Inf. 140(1), 310-322 (2010)

40. Navarro, J., Psarrakos, G.: Characterizations based on generalized cumulative residual entropy functions. Commun. Statist. Theory Meth. 46(3), 1247-1260 (2017)

41. Nigmatullin, R., Baleanu, D.: New relationships connecting a class of fractal objects and fractional integrals in space. Fract. Calculus Appl. Analy. 16(4), 911-936 (2013)

42. Nourbakhsh, M., Yari, G.: Weighted Renyi's entropy for lifetime distributions. Commun. Statist.Theory Meth. 46(14), 7085-7098 (2017)

43. Postigo-Boix, M., Agüero, R., Melús-Moreno, J.L.: An alternative procedure to obtain the mortality rate with nonlinear functions: Application to the case of the Spanish population. PLoS ONE 14(10), e0223789 (2019)

44. Psarrakos, G., Navarro, J.: Generalized cumulative residual entropy and record values. Metrika 76(5), 623-640 (2013)

45. Psarrakos, G., Toomaj, A.: On the generalized cumulative residual entropy with applications in actuarial science. J. Comput. Appl. Math. 309, 186-199 (2017)

46. Rao, M., Chen, Y., Vemuri, B.C., Wang, F.: Cumulative residual entropy: a new measure of information. IEEE Trans. Inf. Theory 50(6), 1220-1228 (2004) 
47. Reed, L.J., Merrell, M.: A short method for constructing an abridged life table. Am. J. Epidemiol. 141(11), 993-1022 (1995)

48. Sachlas, A., Papaioannou, T.: Residual and past entropy in actuarial science and survival models. Methodol. Comput. Appl. Prob. 16(1), 79-99 (2014)

49. Saikia, P., Borah, M.: A study on mathematical modelling for oldest-old mortality rates. Int. J. Mathem. Archive (IJMA) 4(11), 1-9 (2013)

50. Shannon, C.E.: A mathematical theory of communication. Bell Syst. Tech. J. 27(3), 379-423, 623-656 (1948)

51. Shannon, C.E.: A mathematical theory of communication. Bell Syst. Tech. J. 27(3), 379-423 (1948)

52. Sinha, A., Rastogi, S.K., et al.: Inequality in access to improved water source: A regional analysis by Theil Index. Theoretical Economics Letters 5(06), 683 (2015)

53. Smits, J., Monden, C.: Length of life inequality around the globe. Soc. Sci. Med. 68(6), 1114-1123 (2009)

54. Tarasov, V.E.: Lattice model with power-law spatial dispersion for fractional elasticity. Cent. Eur. J. Phys. 11(11), 1580-1588 (2013)

55. Toomaj, A., Di Crescenzo, A.: Generalized entropies, variance and applications. Entropy 22(6), 709 (2020)

56. Van Raalte, A.A., Caswell, H.: Perturbation analysis of indices of lifespan variability. Demography 50(5), 16151640 (2013)
57. Vaupel, J.W.: How change in age-specific mortality affects life expectancy. Populat. Stud. 40(1), 147-157 (1986)

58. Vaupel, J.W., Romo, V.C.: Decomposing change in life expectancy: a bouquet of formulas in honor of Nathan Keyfitz's 90th birthday. Demography 40(2), 201-216 (2003)

59. Wang, F., Vemuri, B.C.: Non-rigid multi-modal image registration using cross-cumulative residual entropy. Int. J. Comput. Vision 74(2), 201-215 (2007)

60. Wilmoth, J.R., Horiuchi, S.: Rectangularization revisited: variability of age at death within human populations. Demography 36(4), 475-495 (1999)

61. Wong, C.H., Tsui, A.K.: Forecasting life expectancy: evidence from a new survival function. Insu. Mathem. Econ. 65, 208-226 (2015)

62. Zhang, Z., Vaupel, J.W.: The age separating early deaths from late deaths. Demograp. Res. 20, 721-730 (2009)

63. Ziegel, E.R.: Survival analysis: a self-learning text. Technometrics 48(2), 317 (2006)

Publisher's Note Springer Nature remains neutral with regard to jurisdictional claims in published maps and institutional affiliations. 